6 Goitein KJ, Amit Y, Mussaffi H. Intracranial pressure in central nervous system infections and cerebral ischaemia of infancy. Arch Dis Child 1983;58:184-6.

Correspondence to Dr M I Levene, Department of Child Health, Clinical Sciences Building, Leicester Royal Infirmary, PO Box 65, Leicester LE2 7LX.

\section{Addendum}

To date this procedure has been successfully performed in 9 infants with no recognised complications. Neither necropsy nor computed tomography in survivors have shown trauma related to the catheter.

\title{
Intrauterine hydrops caused by premature closure of the foramen ovale
}

\author{
E PESONEN, H HAAVISTO, P ÄMMÄLÄ, AND K TERAMO \\ Children's Hospital and the Departments I and II of Obstetrics and Gynecology, University of Helsinki, \\ Finland
}

SUMMARY Intrauterine hydrops was diagnosed by two dimensional echocardiography. The fetus had a pericardial effusion and a thick interatrial septum without a foramen ovale flap. The condition was treated by giving the mother digitalis. Postnatally, the effusion had disappeared and a parachute mitral valve was found.

Fetal hydrops may be reliably diagnosed by ultrasonography. It is often serious and is associated with a high perinatal mortality rate, ${ }^{1}$ but fetal death rarely occurs before the 28th gestational week. Cardiac causes of fetal hydrops include congenital heart diseases and rhythm disturbances. With two dimensional ultrasonography the anatomy of the heart may be visualised ${ }^{2}$ and some idea of the cardiac rhythm obtained. We describe a fetus in whom the foramen ovale was found to be closed on antenatal ultrasound examination: the condition was associated with severe fetal hydrops and an irregular heart rhythm.

\section{Case report}

The mother was a healthy gravida I, para 0 with no family history of cardiac disease. Polyhydramnios was diagnosed by ultrasound on week 31 of gestation. The fetus was hydropic and had ascites. When fetal cardiac ultrasonography was performed on week 32 of gestation, the heart was enlarged and there was fluid in the pericardial cavity (Fig. 1), the interatrial septum was thick and bulged towards the right atrium, the foramen ovale and its valve were not seen and the movement of the atrioventricular valves was irregular. Fetal heart rate monitoring by cardiotocography was attempted unsuccessfully several times and the failure was considered to be caused by irregular heart rate. The fetus was thought to be in cardiac failure and the mother was given digitalis $7 \mu \mathrm{g} / \mathrm{kg} /$ day.

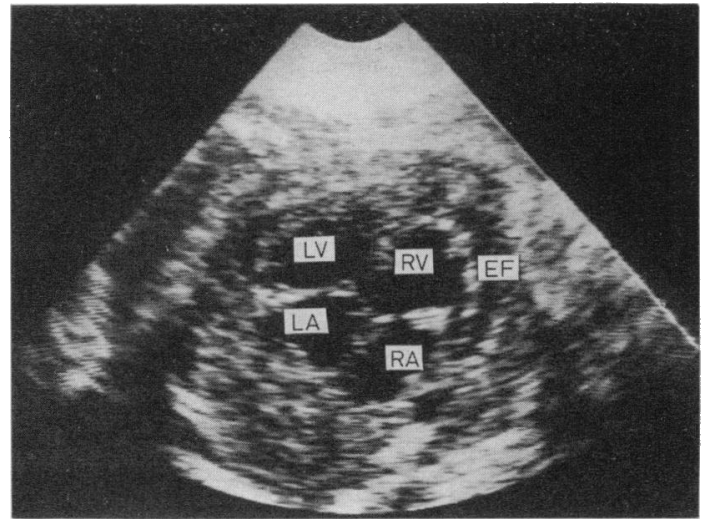

Fig. 1 2-D antenatal echocardiography showing fluid in the pericardial cavity.

$\mathbf{E F}=$ effusion, $\mathbf{L V}=$ left ventricle, $\mathbf{L A}=$ left atrium, $\mathbf{R V}=$ right ventricle, $\mathbf{R A}=$ right atrium.

A boy was born by caesarean section on week 34 of gestation. His Apgar score was 6 at 1 and 5 minutes and he was intubated immediately. His face, neck, abdomen, and scrotum were hydropic and the liver edge was palpable $1.5 \mathrm{~cm}$ below the costal margin. The femoral and brachial arterial pulses were palpable and irregular and on auscultation the second heart sound was not split. A 3/6 systolic heart murmur was heard at the middle left sternal border radiating to the axilla. His electrocardiogram showed three distinct rhythmical morphologies with regular repetition, and the $T$ waves were flat in the left precordial and limb leads suggesting pericardial irritation. Postnatal echocardiography showed no pericardial fluid, the atrial septum was thick and bulging to the right, and there was no evidence of the foramen ovale and its flap in the atrial septum. Mitral valve movement was limited and the chordae tendinae were short and attached to a single large 


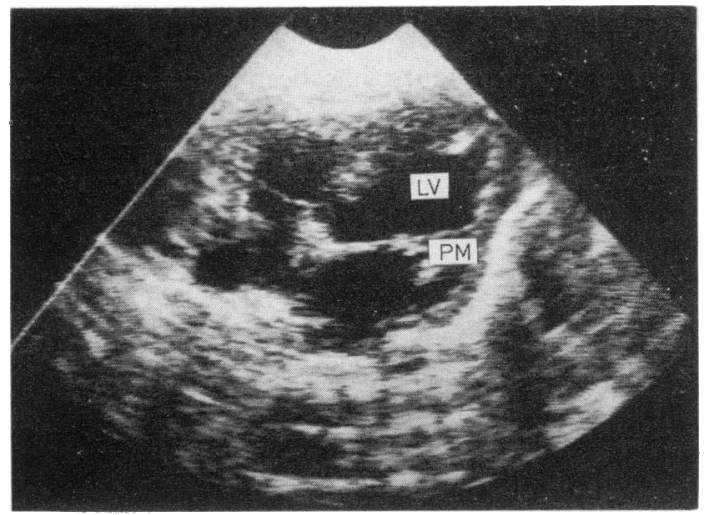

Fig. 2 A postnatal echocardiogram showing the parachute mitral valve deformity.

$\mathbf{P M}=$ papillary muscle, $\mathbf{L V}=$ left ventricle.

papillary muscle (Fig. 2). On the $\mathrm{M}$ mode echocardiogram the ratio of the left atrial to,aortic systolic diameter was $2 \cdot 2$ (upper limit of normal $1 \cdot 3$ ). The EF slope of the mitral valve was flat suggesting slow ventricular filling. The right ventricular systolic time intervals showed a long pre-ejection period (PEP) to ejection time (ET) ratio. This ratio remained high during the first days $(0.61-0.63)$ indicating pulmonary hypertension. ${ }^{3}$ The echocardiographic findings were consistent with parachute mitral valve associated with a thick interatrial septum, closed foramen ovale, and pulmonary hypertension.

At birth the serum digitalis value of cord blood was $0.8 \mathrm{nmol} / \mathrm{l}$. The rhythm was persistently irregular and single unifocal ventricular extrasystoles were noted. During the first 14 days the serum digitalis value increased to $2.6 \mathrm{nmol} / 1$, which is at the upper range of normal. Although the digitalis treatment was stopped, the rhythm did not become normal.

At the age of 5 weeks the infant was thriving moderately well. His chest radiograph still showed pulmonary congestion, the heart was enlarged, but the right ventricular systolic time intervals had returned to normal (PEP:ET $=0 \cdot 21$ ).

\section{Discussion}

Both bradyarrhythmias and tachyarrhythmias may cause fetal hydrops. An irregular fetal heart rate may indicate atrial fibrillation and atrial flutter with variable AV conduction. The treatment of supraventricular atrial tachycardia, atrial fibrillation, and flutter is the same: giving the mother digitalis. Our patient may have had a wandering pacemaker and an irregular heart rhythm in utero.

Severe congenital heart disease may cause antenatal heart failure and fetal hydrops. ${ }^{1}$ To our knowledge only one case of an obstructed foramen ovale, diagnosed antenatally, has been published. ${ }^{4}$ As in our patient increased echoes in the region of the foramen ovale were seen, the patient was oedematous, and pleural effusion was detected.

We presume that in our patient the left atrial pressure was raised in utero. The bulging of the interatrial septum to the right was a sign of a reversal from an intrauterine higher right atrial pressure. A parachute mitral valve caused obstruction to the mitral flow and the increased left atrial pressure sealed the foramen ovale flap. Flat $T$ waves in the electrocardiogram, taken immediately after birth, may have been caused by transient ischaemic subendocardial damage or the recovery phase of myocarditis. Thickening of the atrial septum may be a natural part of the sequela of the intrauterine myocarditis. Interestingly, the patient in the previous report also had a thickened interatrial septum. ${ }^{4}$

If the foramen ovale is closed in utero almost all of the cardiac output has to pass through the right ventricle. This may initiate severe right heart failure and the development of fetal hydrops. When the whole cardiac output is squeezed through the main pulmonary artery, it may increase the muscularity of pulmonary arterioles, causing high pulmonary vascular resistance immediately after birth, ${ }^{5}$ but a few days later the symptoms begin to subside. At the age of 5 weeks our patient still had considerable pulmonary congestion, but the systolic time intervals on the right ventricle had become normal.

It is possible to diagnose cardiac causes of intrauterine hydrops by fetal echocardiography, and giving the mother digitalis may relieve the condition. The prenatal diagnosis and treatment should be carried out by collaboration between obstetricians and paediatric cardiologists.

\section{References}

1 Perlin BM, Pomerance JJ, Schifrin BS. Nonimmunologic hydrops fetalis. Obstet Gynecol 1981 ;57:584-8.

2 Allan LD, Tynan MJ, Campell S, Wilkinson JL, Anderson RH. Echocardiographic and anatomical correlates in the fetus. Br Heart J 1980;44:444-51.

3 Riggs T, Hirschfeld S, Bormuth C, Fanaroff A, Liebman J. Neonatal circulatory changes: an echocardiographic study. Pediatrics 1977;59:338-44.

- Redel DA, Hansman M. Fetal obstruction of the foramen ovale detected by two-dimensional doppler echocardiography. In: Rijsterborgh $\mathrm{H}$, ed. Echocardiology. The Hague: Martinus Nijhoff Publishers, 1981 :425-9.

- Rudolph AM. High pulmonary vascular resistance after birth. 1. Pathophysiologic consideration and etiologic classification. Clin Pediatr (Phila) 1980;19:585-90.

Correspondence to Dr E Pesonen, Children's Hospital, University of Helsinki, Finland.

Received 30 June 1983 